\title{
CAMBIOS TEMPORALES DE LA LLUVIA EN EL OCCIDENTE DE LA ISLA DE CUBA ${ }^{1}$
}

\author{
Teresa López Deulofeu*, José Evelio Gutiérrez Hernández* y Gladis Cabanas Gómez** \\ (*) Grupo de investigaciones Hidroclimatología y Manejo de Cuencas, Facultad de Geografía, \\ Universidad de La Habana \\ (**) Facultad de Matemáticas y Computación, Universidad de La Habana
}

\begin{abstract}
RESUMEN
El presente trabajo surge de la necesidad de conocer en el tiempo, el comportamiento de la lluvia, asi como también en su distribución espacial en el occidente de Cuba. Se analiza la estacionalidad, tendencia, variabilidad y ciclícidad. Se comprobó que por lo menos en el período 1961-1993, no existe diferencia de las estaciones lluviosa y poco lluviosa. Se encontró que la ruptura de la tendencia ocurrió fuertemente en 1975, y una menor en 1973. Se han manifestado 4 ciclos aperíodicos. Se manifiesta el mismo comportamiento de la lluvia en todo el occidente de Cuba.
\end{abstract}

Palabras clave: lluvia, distribución espacial y temporal, estacionalidad, tendencia, variabilidad y ciclicidad.

\begin{abstract}
The main objective of the research was the study of the behavior of rainfall in space and time in the western part of Cuba. Analysis was done in relation to seasonality, trends, variability and the presence of cycles. It was identified that in the period 1961-1993 there was not any displacement of the rainy and dry season. It was identified that this trend was strongly broken in 1975 and in a lesser scale en 1973. Four non periodic cycles were manifested. There is a similar behavior of rainfall in the western part of Cuba.
\end{abstract}

Key words: rainfall, space and temporally distribution, seasonality, trends, variability and rainfall cycles.

1 Este artículo se enmarca en el Programa Cambios Globales y evolución del medio ambiente. Suprograma El ciclo Hidrológico. Proyecto: Antropización en las cuencas hidrológicas del occidente de Cuba y la evaluación de la modificación del ciclo hidrológico. No. 01308031. Ministerio de Ciencia, Tecnología y Medio ambiente. Investigador principal: Dr. Iván González Piedra. 


\section{Introducción}

La lluvia ha sido una de las variables hidroclimáticas más estudiada en Cuba, no obstante, resulta novedoso conocer la presencia, las causas y las consecuencias de los cambios temporales de la lluvia para la interpretación de los regímenes a mediano y largo plazo y su relación con las transformaciones ocurridas en el medio geográfico.

El presente trabajo surge de la necesidad de conocer el comportamiento temporal de la lluvia en el occidente de Cuba, región donde es más variable el tiempo atmosférico, así como su distribución espacial. En él se aplican los métodos de las series cronológicas, cuyo uso nos permite conocer las tendencias en el tiempo de la variable lluvia.

\section{Materiales y métodos}

Los materiales utilizados fueron los datos de lluvia facilitados por el Instituto de Recursos Hidráulicos y el Instituto de Meteorología de Cuba (ISMET) perteneciente al Ministerio de Ciencias, Tecnología y Medio Ambiente (CITMA). Se seleccionaron 20 pluviómetros (tabla 1), los que se identifican con las iniciales de las provincias y el número de asignación que tiene el equipo. Así, Pr (Pinar del Río), Ch (Ciudad de La Habana), Lh (Prov. de La Habana) y Mt (Prov. de La Habana). La mayoría de ellos incluye el período 1961-1990, es decir, el último período climatológico internacional. La región occidental de Cuba abarca las provincias de Pinar del Río, Ciudad de La Habana, La Habana y Matanzas. (Mapa 1),

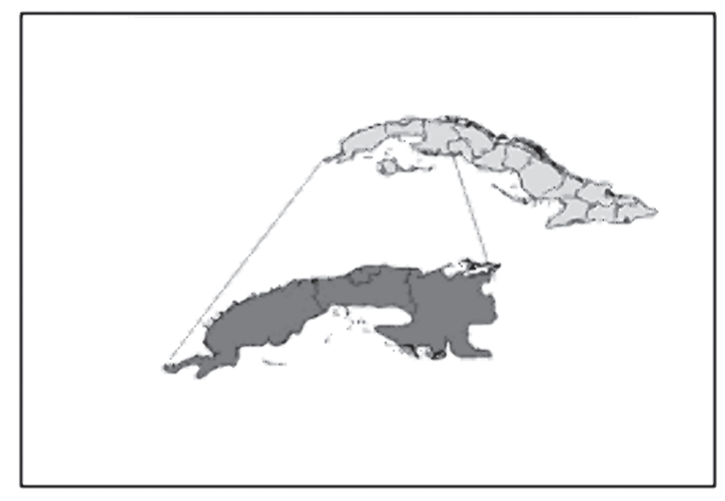

MAPA 1. Región occidental de la Isla de Cuba.

El trabajo se fundamenta en análisis de los resultados de la elaboración estadística y de los gráficos obtenidos en cada uno de los pluviómetros estudiados. Se analiza en detalle para encontrar las rupturas de las tendencias los datos de Casablanca (Ch144), serie que cubre casi todo el siglo XX (1909-2000). En el caso del análisis de la ciclicidad, se utilizaron los pluviómetros Pr4, Pr114, Ch144, Ch15, Lh54, Lh123 y Mt21, para que cada provincia esté representada en dicho análisis (tabla 1). Se destaca en el trabajo la importancia del análisis geográfico de los resultados estadísticos para la aceptación o no de los cambios observados. 
Tabla 1

CARACTERÍSTICAS DE LOS PLUVIÓMETROS SELECCIONADOS

\begin{tabular}{|c|cc|c|c|c|}
\hline Num. pluv & \multicolumn{2}{|c|}{$\begin{array}{c}\text { Coordenada } \\
\text { N }\end{array}$} & $\begin{array}{c}\text { H } \\
\text { (msnm) }\end{array}$ & $\begin{array}{c}\text { Períodos } \\
\text { De observación }\end{array}$ & Num. Años \\
\hline Pr4 & 318,3 & 236,7 & 150 & $1961-1992$ & 32 \\
\hline Pr114 & 304,4 & 228,4 & 55 & $1960-1992$ & 32 \\
\hline Pr119 & 305,4 & 262,7 & 40 & $1950-1992$ & 42 \\
\hline Pr155 & 319,4 & 251,0 & 115 & $1962-1992$ & 30 \\
\hline Pr157 & 217,4 & 232,3 & 140 & $1962-1992$ & 31 \\
\hline Pr159 & 319,3 & 239,3 & 130 & $1963-1992$ & 30 \\
\hline Pr161 & 317,4 & 252,9 & 100 & $1962-1992$ & 31 \\
\hline Ch12 & 351,1 & 351,9 & 70 & $1952-2000$ & 48 \\
\hline Ch15 & 350,4 & 357,2 & 76 & $1907-2001$ & 95 \\
\hline Ch144 & 368,7 & 362,5 & 50 & $1909-2000$ & 91 \\
\hline LH 54 & 368,6 & 393,1 & 20 & $1931-1999$ & 68 \\
\hline Lh123 & 357,2 & 414,0 & 75 & $1940-1994$ & 54 \\
\hline Lh410 & 359,4 & 421,8 & 110 & $1964-1993$ & 30 \\
\hline Lh420 & 354,5 & 427,2 & 80 & $1964-1993$ & 30 \\
\hline Lh421 & 360,0 & 429,1 & 70 & $1932-1993$ & 61 \\
\hline Lh423 & 346,0 & 423,9 & 130 & $1964-1993$ & 33 \\
\hline Mt21 & 338,1 & 436,9 & 160 & $1928-1991$ & 63 \\
\hline Mt289 & 350,3 & 431,9 & 36 & $1961-1991$ & 31 \\
\hline Mt376 & 350,3 & 245,9 & 75 & $1966-1991$ & 26 \\
\hline Mt404 & 354,3 & 434,6 & 12 & $1966-1991$ & 26 \\
\hline
\end{tabular}

Fuente: Expediente de los pluviómetros. Instituto Recursos Hidráulicos. La Habana. Cuba.

El método que se utilizó para el análisis estadístico fue el de series cronológicas. Este método facilita conocer las causas de los movimientos experimentados en una serie de tiempo, y se conocen con el nombre de componentes, que son: las variaciones estacionales, la tendencia, la ciclicidad y la componente aleatoria. El modelo que relaciona estas componente es el aditivo, pues corresponde al de mejor ajuste (Cansado, 1966; Wei, 1990; Willks, 1995; Freund, 1977).

Las variaciones estacionales: Dan los movimientos periódicos que se producen en el transcurso de un año. Su cálculo tiene dos fases:

- comprobar su existencia significativa

- estimar los patrones estacionales 
La tendencia: Es el movimiento suave y continuo durante un período de tiempo por una causa mantenida. Para determinar la tendencia se parte de dos procedimientos:

- ajuste de una línea

- medias móviles

Las variaciones estacionales y la tendencia son movimientos determinísticos y se calculan de forma simultánea según el modelo supuesto aditivo, cuya estimación descansa en las técnicas de los mínimos cuadrados ordinarios, utilizándose los promedios de las medias móviles en la suavización de las series.

En el caso de la estacionalidad se da una metodología para, con el uso de modelos regresivos, detectar un cambio estable en el patrón estacional de esta variable, y las pruebas de hipótesis correspondientes. La componente estacional representa cambios a través de los años, pero en una forma lenta y progresiva, para lo que se utilizó la estación de Casablanca, que posee la serie de datos más larga.

La tendencia puede tener rupturas que responden a cambios reales en la misma o a que se recogen dos movimientos diferentes dentro de un ciclo. Para diferenciar estos dos fenómenos, e incluso para detectar la existencia de la ruptura en una variable con una desviación típica tan grande, también hace falta tener una longitud considerable en la serie de las observaciones. En el trabajo se dan diferentes pruebas para comprobar la posible presencia y/o ruptura de la tendencia incluyendo como más recomendable en este caso la prueba de Kendall Mann, discutiéndose sus limitaciones.

Las variaciones cíclicas o la ciclicidad son los movimientos oscilatorios que se producen en el transcurso del tiempo. La ciclicidad se obtiene por las curvas integrales de los coeficientes modulares: $(\mathrm{CICM}), \Sigma \mathrm{K}-1 / \mathrm{CV}=\Sigma$ (f) $\mathrm{t}$ años, para conocer las variaciones aperiódicas y para las periódicas se analizan los gráficos de Periodogramas.

El procesamiento del análisis se realizó mediante: el paquete estadístico Statgraphic, versión 5.0 STSC and Statistical Graphic corporation 1985-1991, y el EXCEL sobre Window, donde se guardaron los bases de datos, además de varios paquetes estadísticos comerciales como el Sgwin y el Eviw.

\section{Análisis de los resultados}

\subsection{Variaciones estacionales}

Para conocer la significación de la estacionalidad o comportamiento de los valores mensuales dentro del año cronológico, se analizaron todos los pluviómetros y se obtuvieron una serie de gráficos de los que a manera de ejemplo sólo se presentan en el texto los de los pluviómetros Pr4 y Ch144, con las figuras de los periodogramas, los periodogramas integrados y el índice de estacionalidad (Figs. 1, 1a y 2, 2a). además de las componentes estacionales estimadas y residuales (Figs 3, 3a y 4, 4a).

Por análisis de las figuras, la estacionalidad resultó ser significativa, su distribución es bimodal, presenta un máximo primario en el mes de junio y otro secundario en septiembre, en la mayoría de los pluviómetros. Los valores mínimos se observan en los meses de enero, febrero, marzo, abril, noviembre y diciembre. En Pinar del río los meses de menor cantidad de lluvia resultaron diciembre y enero y en Matanzas no están uniforme, ya que los distintos pluviómetros, los valores mínimos se estiendoen hasta abril.

Existe una diferencia entre los pluviómetros situados cerca de la costa y los que se encuentran ubicados a $5 \mathrm{~km}$. aproximadamente de ésta. Los situados cerca de la costa 


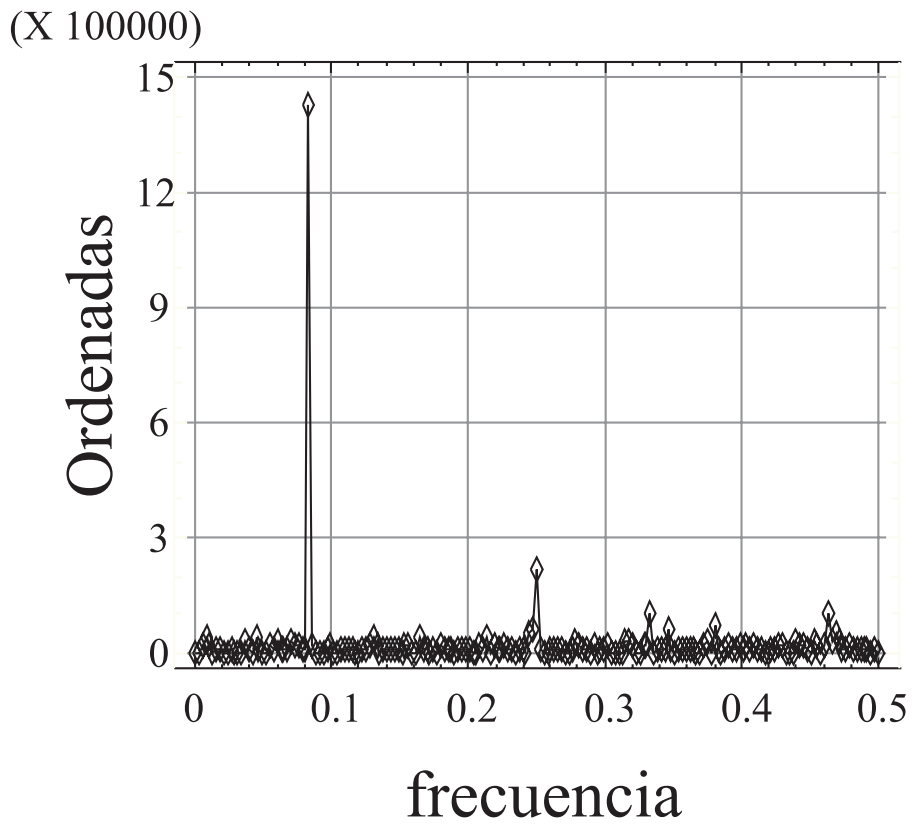

Figura 1. Periodograma para la lluvia. Pr4.

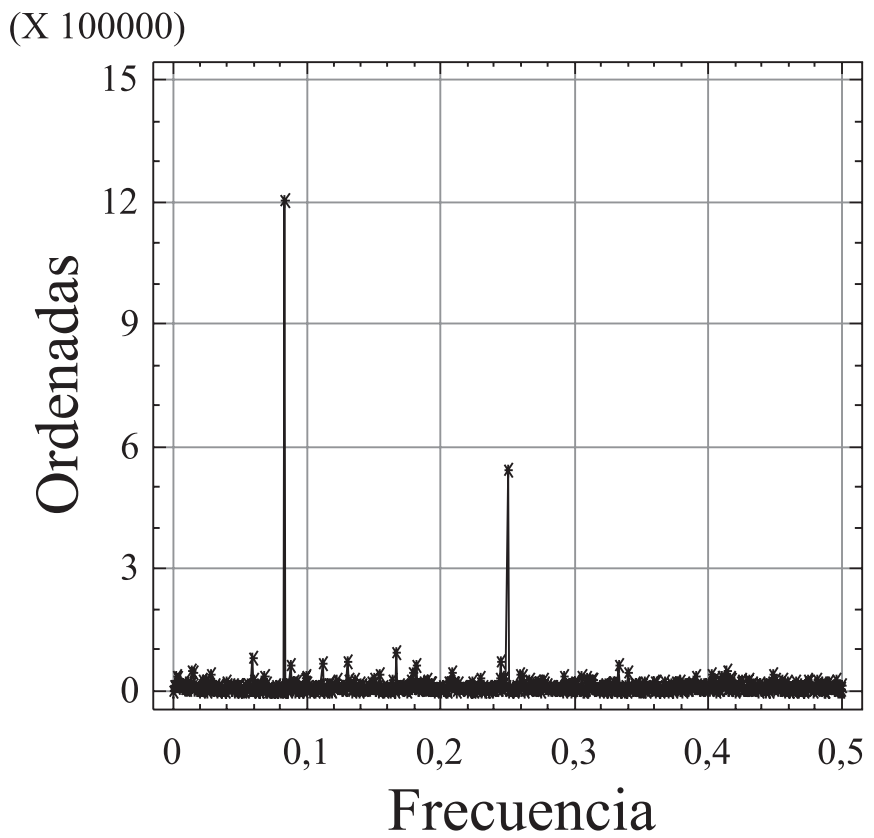

FIgURA 1a. Periodograma para la lluvia. Ch144. 


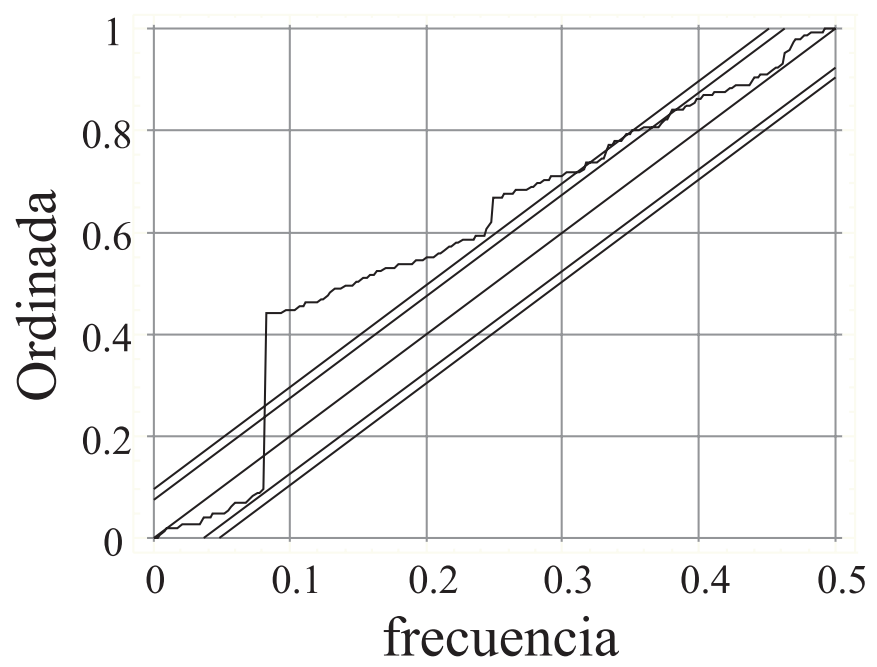

FiguRa 2. Periodograma Integrado para la lluvia. Pr4.

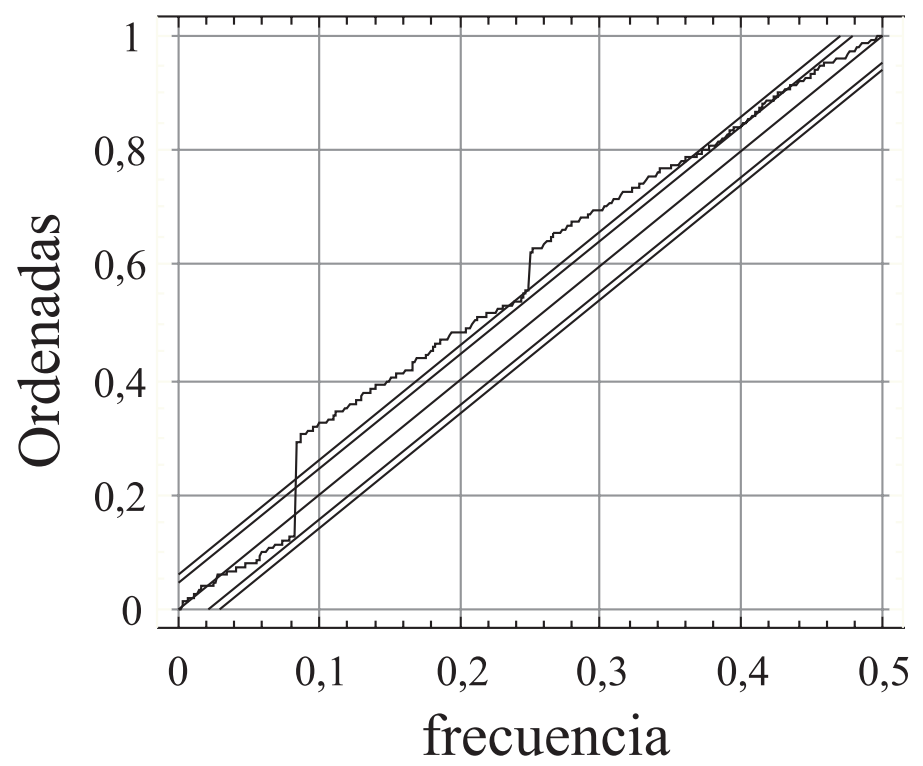

Figura 2a. Periodograma Integrado para la lluvia. Ch144. 


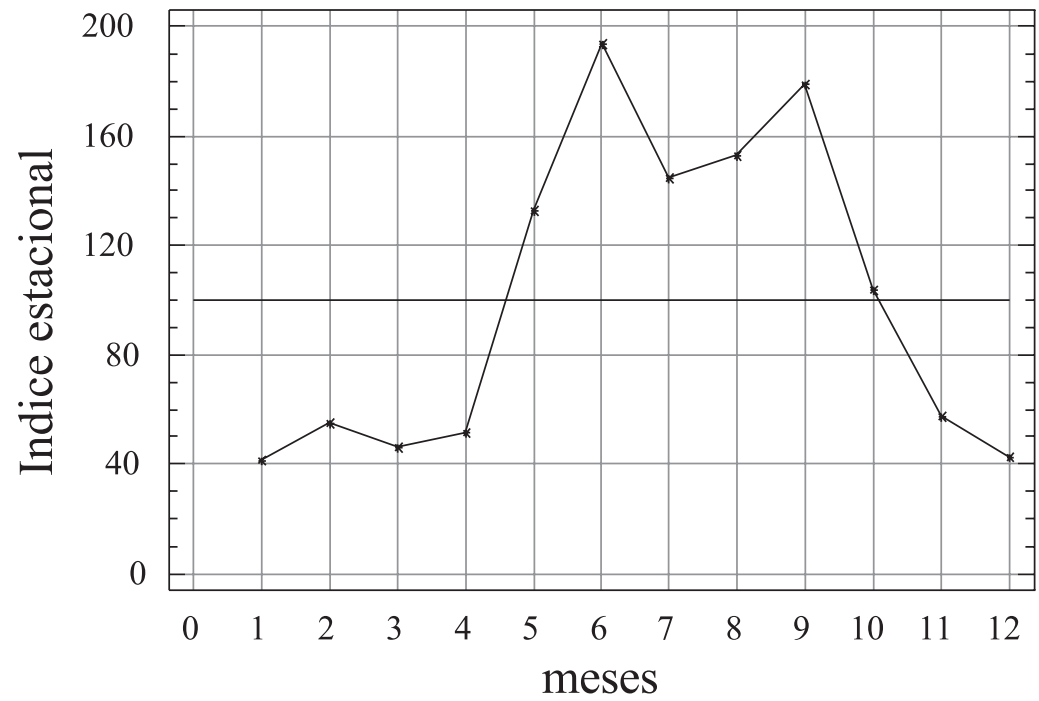

FIgURA 3. Índice estacional para la lluvia. Pr4.

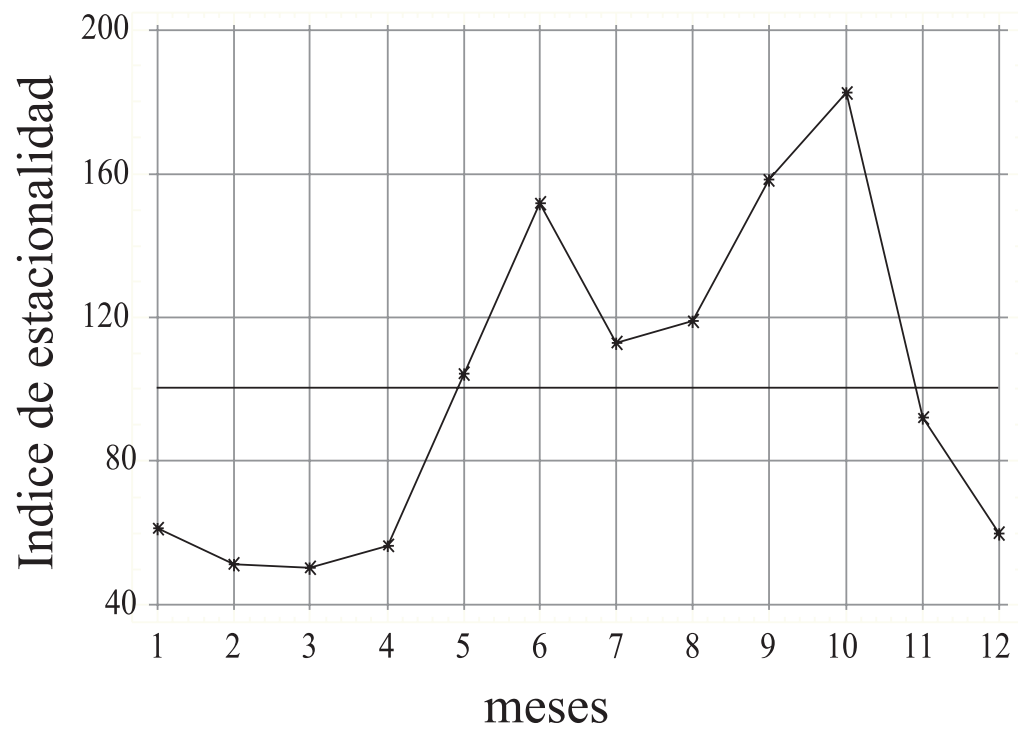

FIGURA 3a. Índice de estacionalidad para Ch144. 


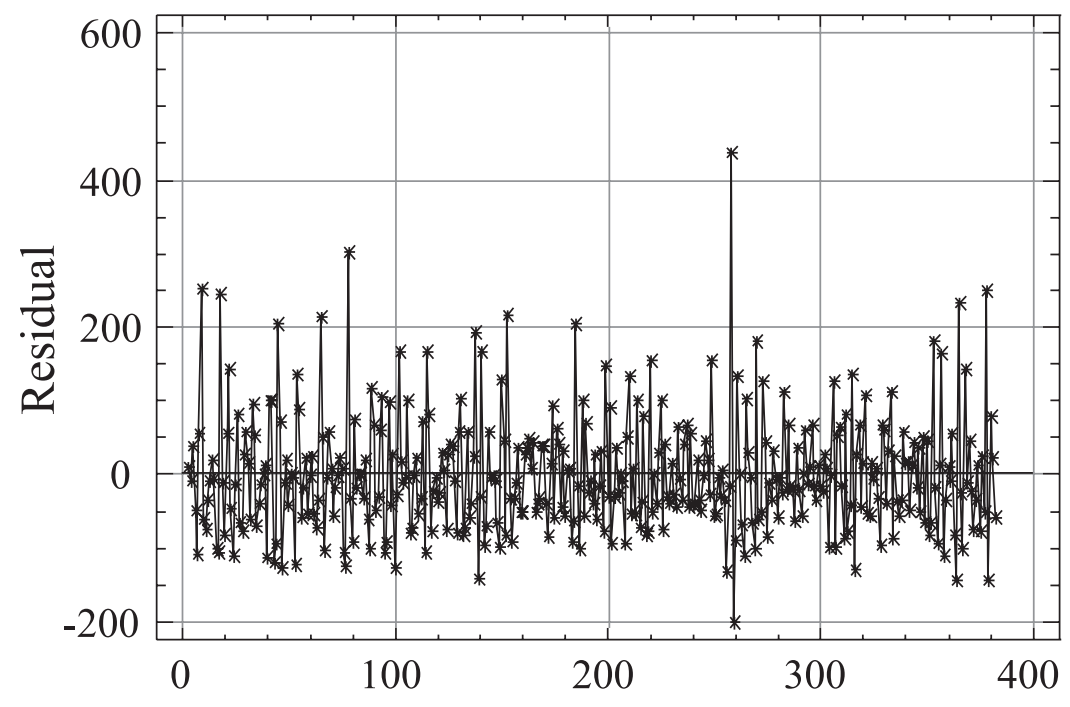

Figura 4. Residuales de la lluvia. Pr4.

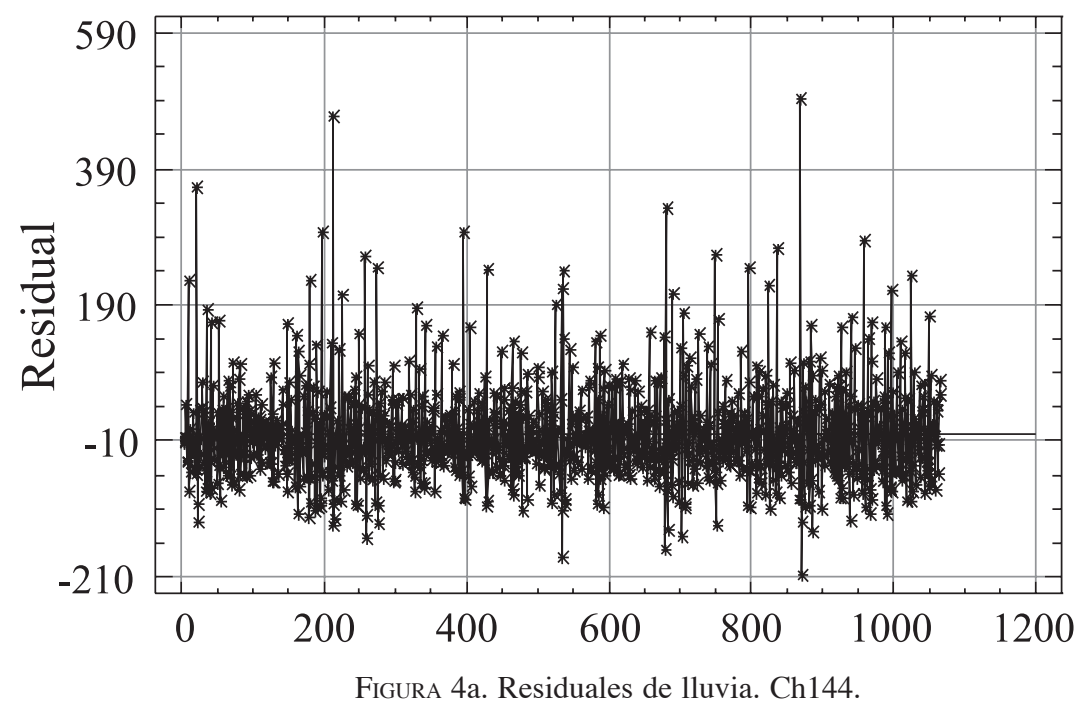


presentan el máximo primario o principal en octubre y el secundario en junio. La causa de esta distribución de la lluvia es que hacia el interior la convección se acelera a partir de mayo, alcanzando la mayor intensidad en junio; no ocurre lo mismo cerca de la costa, ya que no es hasta octubre que los mares alcanzan su mayor temperatura y es más frecuente la presencia de nubes de lluvias.

Los valores mínimos se concentran en los meses de marzo y abril. Además la sequía intraestival, o sea la disminución de la lluvia en los meses de julio y agosto, presenta valores más bajos cerca de la costa que hacia el interior del territorio.

Existe una diferencia entre los pluviómetros situados cerca de la costa y los que se encuentran ubicados a $5 \mathrm{~km}$. aproximadamente de ésta. Los situados cerca de la costa presentan el máximo primario o principal en octubre y el secundario en junio. La causa de esta distribución de la lluvia es que hacia el interior la convección se acelera a partir de mayo, alcanzando la mayor intensidad en junio; no ocurre lo mismo cerca de la costa, ya que no es hasta octubre que los mares alcanzan su mayor temperatura y es más frecuente la presencia de nubes de lluvias.

Los valores mínimos se concentran en los meses de marzo y abril. Además la sequía intraestival, o sea la disminución de la lluvia en los meses de julio y agosto, presenta valores más bajos cerca de la costa que hacia el interior del territorio.

\subsection{Variabilidad}

Como se puede observar en el ejemplo de las figs. 5 y $5 \mathrm{a}$, la variabilidad es muy alta en los meses del período lluvioso, sobre todo en mayo, junio, julio y septiembre, y en algunos pluviómetros octubre y noviembre. En el período poco lluvioso la variabilidad disminuye desde diciembre a abril No se detecta que sea sistemático que los valores mayores de variabilidad se acumulen ni en los primeros ni en los últimos años del período estudiado.

Por el análisis de los datos de la lluvia mensual en el período estudiado se evidenció que los valores de lluvia de los meses de febrero y marzo han aumentado en los últimos años con relación a su comportamiento normal. También destaca en los análisis de la variación estacional y de la tendencia que en los meses de junio, septiembre y octubre se dio una disminución.

Para darle explicación a este comportamiento de la lluvia, se consultó las condiciones atmosféricas que pudiesen haber influido en la lluvia en el período estudiado.

A partir de la información contenida en el «Resumen de temporada ciclónica», Departamento de Pronóstico, Instituto de Meteorología (ISMET) del Ministerio de Ciencias, Tecnología y Medio Ambiente (CITMA), de Mapas Sinópticos, y material de archivo, se elaboró el cuadro 2. En él se observa que el período de estudio se presentó con poca actividad ciclónica, y que en el mes de octubre, a pesar del aumento del numero de ciclones en la última década, éstos no pasaron de las categorías de depresión y tormenta tropical. En dos de ellos, las lluvias no sobrepasaron los $70 \mathrm{~mm}$ y en los restantes no alcanzaron los $170 \mathrm{~mm}$, es decir, que en general la actividad ciclónica disminuyó su influencia sobre Cuba en el período 1961-1990.

Por el análisis de los gráficos de la tendencia de cada mes (ver ejemplos en las figs. 6 y 7), que se tratarán más detenidamente en el epígrafe siguiente, se supuso que había una estacionalidad dinámica con cambios de los índices de los meses de febrero y marzo, es decir, que estos meses parecen estar cambiando sus valores promedios. 


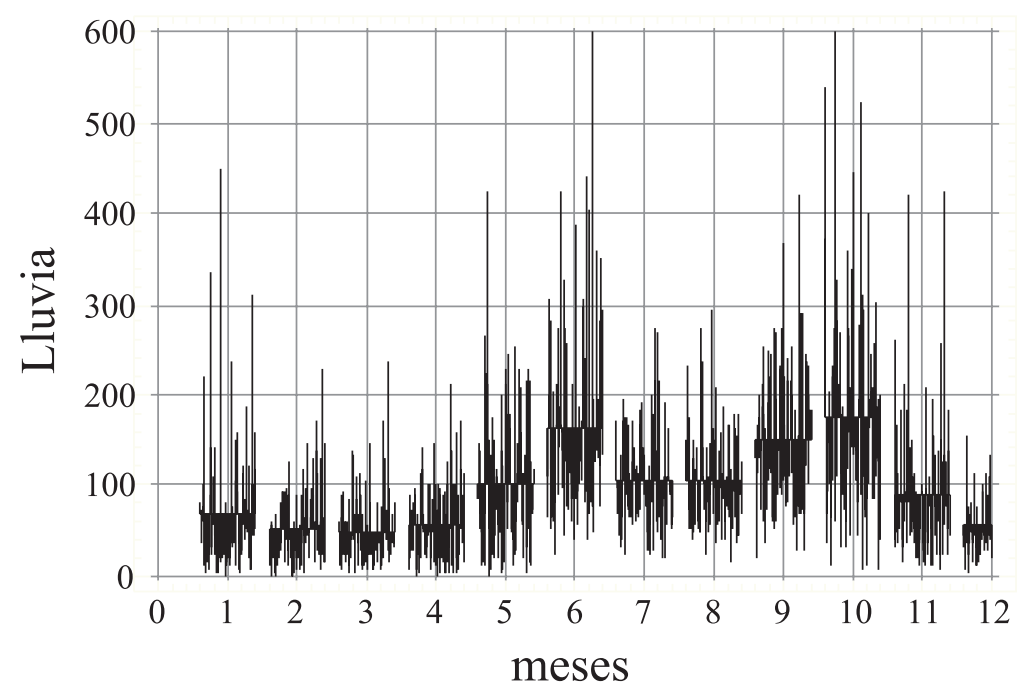

Figura 5. Variabilidad de la lluvia. Pr 4.

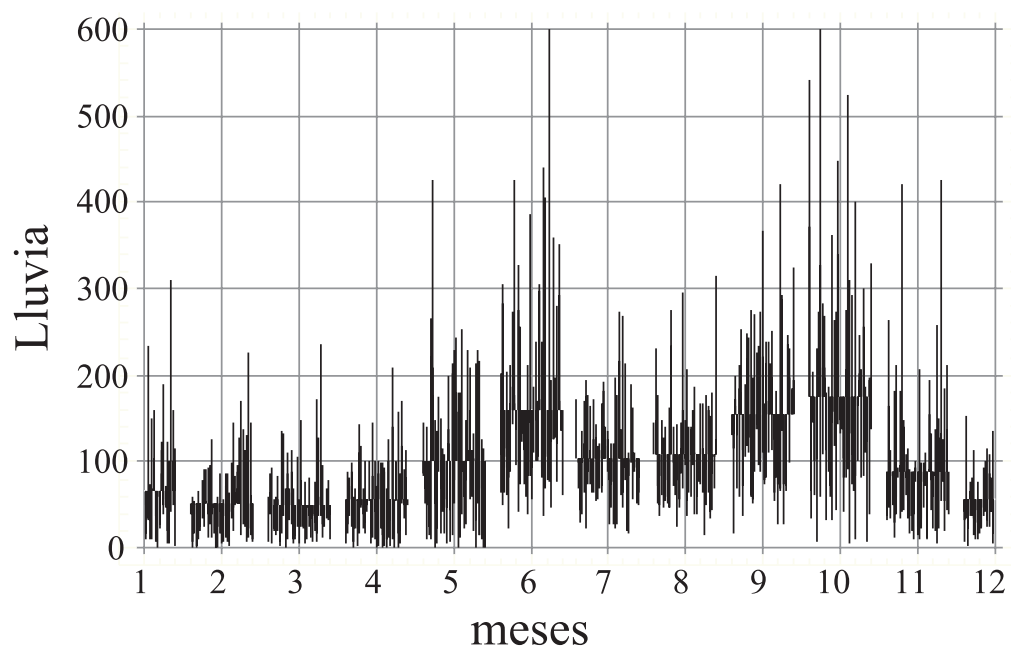

FIgURA 5a. Variabilidad de la lluvia. Ch144. 
Tabla 2

CICLONES QUE HAN AFECTADO A CUBA EN LA REGIÓN OCCIDENTAL EN LAS DÉCADAS DEL PERÍODO 1961-1990

\begin{tabular}{|c|c|c|c|}
\hline Décadas/ meses & Junio & Sept. & Oct \\
\hline $1961-1970$ & 3 & 3 & 3 \\
\hline $1971-1980$ & 1 & 2 & 1 \\
\hline $1981-1990$ & 2 & 1 & 4 \\
\hline
\end{tabular}

Fuente: Resumen de la temporada ciclónica. Dpto. de Pronóstico. (ISMET,CITMA). La Habana. Cuba.

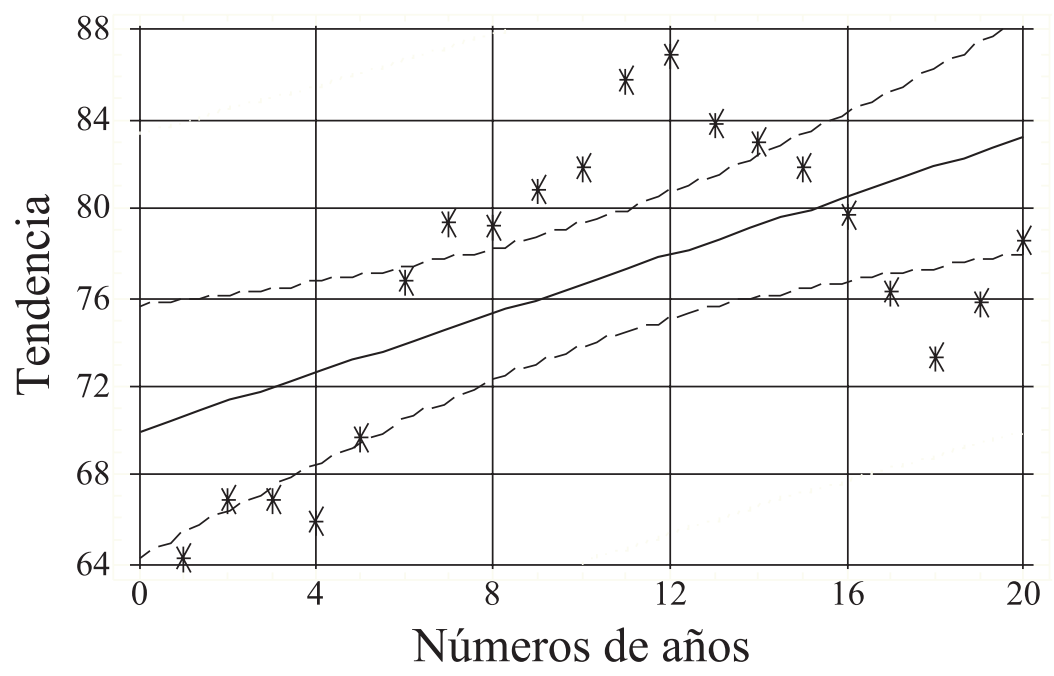

Figura 7. Línea de tendencia. Mes de marzo. Pr4.

Para llegar a la afirmación anterior se compararon las pendientes o tendencias mensuales contra las anuales, en los meses donde eran significativas las mismas, aplicándose una prueba de hipótesis de igualdad de pendiente en las rectas ajustadas por regresión.

Para conocer si los meses han experimentado un desplazamiento de su índice estacional se aplicó una prueba binomial, para ver si el número de pluviómetro que presentaba diferencias era significativo. La expresión utilizada para aplicar la prueba es la siguiente: 
$\left|\mathrm{b}_{1}-\mathrm{b}_{2}\right| \mathrm{S}_{\mathrm{N}} \sqrt{N} / \mathrm{S}_{\text {mensual }}>\mathrm{t}(\mathrm{N}-2)$ donde:

$\mathrm{b}_{1}=$ Pendiente de los valores anuales

$\sqrt{N}=$ Raíz cuadrada del número de años

$\mathrm{S}_{\text {mensual }}=$ Desviación estándar del mes

$\mathrm{N}=$ Número de años $\mathrm{b}_{2}=$ Pendiente de los valores mensuales.

$\mathrm{S}_{\mathrm{N}}=$ Desviación estándar de los años

$\mathrm{t}=\mathrm{t}-$ Student

0,95 nivel de confianza.

En la tabla 3 aparecen los valores mensuales calculados para cada pluviómetro, en ella se destaca si las pendientes de las series mensuales difieren de las del año. Para identificarlas se indica dif si hay diferencia, y no dif si no la hay. Se puede observar que en la temporada seca hay un mayor número de pluviómetros con diferencias que en los meses del período húmedo y dentro de aquélla, los meses de febrero y marzo tienen la mayoría con diferencias. Esto puede deberse a que los valores habituales de estos meses son más estables, con lo que cualquier variación, por pequeña que sea, puede resultar significativa. Desde el punto de vista espacial, con un $95 \%$ de confianza, cambian 10 pluviómetros en estos meses, por lo que se asume que en ellos se ha producido un cambio en la cantidad de lluvia recibida.

Este análisis se realizó con el fin de detectar si se están manifestando cambios en los meses que conforman los períodos lluviosos y poco lluviosos o si se está presentando un corrimiento en el año. Esto último no se confirmó y se puede afirmar que en el período fundamental analizado, 1961-1993, no se presenta corrimiento de las épocas de lluvia y poca lluvia, y que sólo se manifiesta que los meses de febrero y marzo están cambiando su patrón estacional (ellos siguen siendo secos, pero la lluvia ha ido aumentado en el período analizado).

Hay que señalar que el lapso de tiempo (1961-1993) resulta ser corto para el análisis del cambio del patrón estacional, ya que cualquier anomalía de peso lo altera. Dentro de este período, específicamente los meses de febrero y marzo fueron afectados por dos eventos de El Niño, uno ocurrido en 1982-1983, (Deser et al, 1987), que fue clasificado por su intensidad como un evento fuerte, y el otro en 1986-1987, cuyas anomalías e intensidad indican la ocurrencia de un evento El Niño de carácter moderado (Carnejo-Rodríguez, 1990).

Por lo antes expuesto, el cambio de patrón estacional de febrero y marzo puede responder al hecho de que dos temporadas invernales de este período fueron afectadas por dos eventos de El Niño.

Las condiciones meteorológicas que influyeron en Cuba en febrero de 1983 y marzo de 1987, según aparece en el resumen de las temporadas invernales del Dpto de Pronóstico, ISMET, CITMA, resultó ser la siguiente:

Febrero 1983. Temporada fuera de lo común, no sólo en Cuba sino también en otras regiones, como, por ejemplo, Ecuador, Bolivia, Perú y Argentina. Para el Golfo de México, La Florida y Cuba se puede clasificar esta temporada como una de las peores del siglo. En Cuba no se presentaba un invierno con características similares desde 1958, pero con la diferencia de que en este año predominaron sobre la lluvia, vientos fuertes y temperaturas bajas. Se mantuvo el mal tiempo debido a un frente frío en el centro del Golfo de México, que produjo fuertes chubascos en la región occidental.

Marzo 1987. La corriente en chorro subtropical se sitúa sobre México y el Golfo de México, provocando en el Caribe y en Cuba el desarrollo de extensas áreas de nublados, lluvia, la intensificación de bandas frontales y la formación de bajas extratropicales en 
Tabla 3

ANÁLISIS DE LAS DIFERENCIAS DE LAS PENDIENTES MENSUALES CONTRA LOS VALORES ANUALES EN CADA PLUVIÓMETRO

\begin{tabular}{|c|c|c|c|c|c|c|c|c|c|c|c|c|}
\hline Pluv & $\mathrm{E}$ & $\mathbf{F}$ & M & A & M & $\mathbf{J}$ & $\mathbf{J}$ & A & $S$ & 0 & $\mathbf{N}$ & D \\
\hline 24 & & $\mathrm{~d}$ & dif & dif & 0 dif & dif & dif & dif & 0 dif & dif & dif & no di \\
\hline 11 & dif & 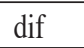 & & & 0 dif & 0 dif & 0 dif & mif & dif & no dif & if & dif \\
\hline 155 & dif & dif & & dif & dif & 0 dif & 0 dif & dif & 10 dif & no dif & no dif & 10 dif \\
\hline PR157 & dif & dif & & & if & if & lif & dif & diff & dif & dif & dif \\
\hline 15 & & & & & & & dif & dif & dif & dif & dif & dif \\
\hline PR161 & dif & 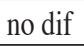 & 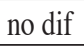 & 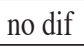 & 11 & 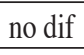 & no dif & $\mathrm{nc}$ & no dif & if & & no dif \\
\hline PR182 & 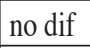 & no dif & dif & dif & 0 dif & dif & no dif & no dif & no dif & no dif & & no dif \\
\hline MT21 & dif & d & & & & dif & dif & no dif & dif & dif & dif & dif \\
\hline MT289 & dif & no di & 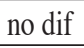 & 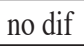 & dif & dif & no dif & no dif & no dif & dif & no dif & no dif \\
\hline MT376 & dif. & 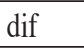 & & & . & $m$ & no dif & no dif & no dif & no dif & dif & dif \\
\hline MT404 & 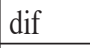 & dif & & & & & dif & & & & & 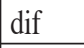 \\
\hline MT409 & dif & dif & & & & & dif & m dif & 0 dif & dif & & \\
\hline LH410 & & nod & & & & no dif & nod & dif & no dif & no dif & di & nod \\
\hline LH420 & & dif & & & & dif & dif & dif & no dif & di & $\mathrm{di}$ & dif \\
\hline LH423 & no dif & no dif & no dif & no dif & no dif & no dif & no dif & no dif & no dif & no dif & no dif & no dif \\
\hline
\end{tabular}

Fuente: Valores calculados por los autores.

latitudes tan bajas como al sur de la Isla de Cuba. La lluvia sobrepasó la normal histórica de 3 a 5 veces desde Pinar del Río a Ciego de Ávila.

En la tabla 4 aparece el resultado del análisis que se efectuó a partir de los gráficos de las series mensuales de todos los pluviómetros, figs. 6 y 7. En la tabla se señala con un signo positivo si la curva asciende y con un signo negativo si desciende, además se añade si es leve (L) o casi recta (CR).

Se destaca que en los meses del período poco lluvioso la lluvia tiene una tendencia a aumentar, y en los meses del período lluvioso a disminuir. Aunque no en todos los pluviómetros las pendientes son significativas, se puede afirmar lo anterior, porque cuando un fenómeno se repite con una alta frecuencia, aunque la pendiente no sea significativa en cada pluviómetro en particular, se debe tomar en cuenta, ya que la significación se la da la repetición.

En los casos de mayor duda (alfa observado entre 0.05 y 0.015 ) se realizó la prueba de Kendall-Mann para la existencia de tendencia, dando en todos los casos existencia de la misma al $90 \%$ de confianza.

Este mismo comportamiento concuerda con las curvas integrales calculadas (curvas de coeficientes modulares, fig. 8), en las que se destaca que hay una tendencia al descenso desde el año 1933 hasta el inicio de la década de 1980. Además también lo corrobora el resultado alcanzado por Lapinel 1997 y por Lora 1999, en el «Estudio preliminar de tendencia de las precipitaciones», donde se analiza una serie de 137 años (1861-1997) del pluviómetro de Belén-Casablanca, y en la mayoría de los años los valores medios anuales están por debajo de la media histórica. En ambos resultados se plantea una recuperación discreta de la lluvia en las dos últimas décadas. 
Tabla 4

RESULTADOS DEL ANÁLISIS DE LA TENDENCIA MENSUAL

\begin{tabular}{|l|l|l|l|l|l|l|l|l|l|l|l|l|}
\hline Pluv & Ene & Feb & Mar & Abr & May & Jun & Jul & Agost & Sept & Oct & Nov & Dic \\
\hline Pr4 & - & + & + & + & + & - & - & + & + & - & + & + \\
\hline Pr114 & - & + & + & + & + & + & + & + & - & - & + & + \\
\hline Pr155 & - & + & + & + & - & - & - & + & - & - & + & + \\
& & & & & & & & & & & & \\
\hline Pr157 & - & + & + & + & - & - & - & + & + & - & + & - \\
\hline Pr159 & + L & + & + & + & CR & - & + L & + L & + & + L & + & + \\
\hline Pr161 & - & + & + & + & + & + & + & + & - & - & + & + \\
\hline Pr182 & - & - L & + & + & + & - & + & - & + L & - & + & + \\
\hline Lh410 & - & - L & + & - & - L & CR & - & + L & + & - & + & + \\
\hline Lh420 & - & + & + & + & + & - & - L & + & + & - & + L & - L \\
\hline Lh423 & + L & - & + & + & + & - L & - L & - & - L & - L & + & + \\
\hline Mt289 & - & + & + & + & + & - & - L & - & - & - & + & - \\
\hline Mt376 & - & + & + & + & + & - & + & - L & - & - & + & + \\
\hline Mt404 & - & + & + & - L & - L & CR & - L & - & CR & - & - & - \\
\hline Mt409 & - & + & + & + & + & - L & - L & - & - & - & - L & + \\
\hline
\end{tabular}

Fuente: Valores calculados por los autores.

Para comprobar exactamente los años en que se produjo la ruptura de la tendencia se le aplicó a la serie de datos del pluviómetro de Casablanca, casi con un siglo de observaciones, un análisis de varianza, cuyos resultados se exponen a continuación:

Las rupturas se produjeron en los años 1973 y 1975, siendo esta última mucho más marcada que la primera. Esto se comprueba también en las curvas integrales, donde se manifiesta que en el año 1975 existe un cambio en las fases de los ciclos (fig. 8).

\subsection{Variaciones cíclicas de los valores mensuales}

De los gráficos de los periodogramas y los periodogramas integrados de cada pluviómetro (figs. 1, 1a y 2, 2a) se puede extraer que hay único ciclo periódico destacable, que es el anual.

En cuanto a los posibles ciclos aperiódicos la metodología de uso de las curvas integrales plantea que debe hacerse con los valores anuales.

\subsection{Componente aleatoria o residual}

Las componentes residuales de cada pluviómetro fueron sometidas a pruebas de normalidad, igualdad de medias (primera mitad de la serie contra segunda mitad) y pruebas de igualdad de varianzas para cada una de las series. A continuación se refleja en la tabla 5 los niveles de confianza con que se aceptaron dichas hipótesis en los tres casos. (fig. 4, 4a). 
Tabla 5

RUPTURA DE LA TENDENCIA

Ruptura en 1973

Análisis de varianza de la lluvia

\begin{tabular}{|c|c|c|c|c|c|}
\hline Causa & Suma de cuad. & GL & C. medios & F-Radio & Nivel de sig. \\
\hline \multicolumn{6}{|l|}{ EFECTOS } \\
\hline A:año & 18939.8 & 1 & 18939.8 & 3.48 & 0.0621 \\
\hline B:mes & $1.94005 \mathrm{E} 6$ & 11 & 176368.0 & 32.42 & 0.0000 \\
\hline RESIDUAL & 5.73992E6 & 1055 & 5440.68 & & \\
\hline TOTAL & 7.6989E6 & 1067 & & & \\
\hline
\end{tabular}

Ruptura en 1975

Análisis de varianza

\begin{tabular}{lrrrrr}
\hline Causa & Suma de cuad. & GL & C. medios & F-Radio & Nivel de sig. \\
\hline EFECTOS & & & & & \\
A:años & 28514.4 & 1 & 28514.4 & 5.25 & 0.0220 \\
B:meses & $1.94005 E 6$ & 11 & 176368.0 & 32.47 & 0.0000 \\
RESIDUAL & $5.73034 E 6$ & 1055 & 5431.61 & & \\
- & & &
\end{tabular}

La tendencia anual es negativa dentro del período 1961-1993 según muestran los datos de la tabla 6, en el que se puede observar que las pendientes no tienen grandes valores, pero en su mayoría son significativas.

Esta tendencia se origina porque en los meses del período lluvioso, sobre todo junio y octubre, predomina en todos los pluviómetros una tendencia negativa (tabla 6). Como el período de estudio es relativamente corto pudiera ser un decrecimiento temporal y no una tendencia duradera, por lo que sólo puede afirmarse que dentro del período de estudio tuvo esta inclinación, aunque hay que ser cauto con los pronósticos.

Tabla 6

NIVELES DE SIGNIFICACIÓN OBSERVADOS

\begin{tabular}{|c|c|c|c|}
\hline & Hondo & San Diego & San Agustín \\
\hline Prueba de Normalización $\%$ & $8,6 \%$ & $4,9 \%$ & $6,2 \%$ \\
\hline Medias & $32 \%$ & $41 \%$ & $25 \%$ \\
\hline Varianza & $21 \%$ & $22 \%$ & $10 \%$ \\
\hline
\end{tabular}

Fuente: Valores calculados por los autores. 


\subsection{Variaciones cíclicas de los valores anuales}

Los periodogramas de las series anuales no reflejaron períodos significativos, lo cual sólo quiere decir que los posibles ciclos existentes en el período no son periódicos. Por este motivo se pasó al estudio de las curvas integrales.

Tabla 7

ANÁLISIS DE REGRESIÓN DE LAS SERIES DE PLUVIÓMETROS SELECCIONADOS

\begin{tabular}{|l|l|l|l|}
\hline Pluv. & Pendiente & Nivel de significación & \\
\hline pr4 & $-0,019$ & 0,06 & \\
\hline pr114 & $-0,025$ & 0,03 & sig \\
\hline pr155 & $-0,015$ & 0,2 & \\
\hline pr157 & $-0,058$ & 0,0005 & sig \\
\hline pr159 & 0,03 & 0,09 & \\
\hline pr182 & $-0,036$ & 0,017 & sig \\
\hline mt289 & $-0,058$ & 0,00001 & sig \\
\hline mt376 & $-0,0169$ & 0,237 & \\
\hline mt404 & $-0,034$ & 0,017 & sig. \\
\hline mt409 & $-0,0258$ & 0,067 & \\
\hline $\operatorname{lh} 410$ & $-0,02$ & 0,037 & sig. \\
\hline $\operatorname{lh} 420$ & 0,031 & 0,005 & sig \\
\hline
\end{tabular}

Fuente: Valores calculados por los autores

\subsection{Ciclos aperiódicos. Curvas integrales}

Los ciclos multianuales permiten destacar los períodos correspondientes a un conjunto de años en que la magnitud de estas variables manifiesta ascensos y descensos sucesivos.

El estudio de esta regularidad requiere un procesamiento estadístico especial de la serie anual de datos, sugerido y empleado en el estudio de series periódicas de diversa naturaleza, consistente en la obtención de una función de distribución suavizada o alisada y estandarizada, que permite su fácil representación esquemática, interpretación y comparación con otros puntos de observación u otras variables afines; de ahí la expresión utilizada y anteriormente expuesta.

Para aplicar el método de las Curvas Integrales de Coeficientes Modulares (CICM) se utilizaron los pluviómetros que contaban con un período largo de observación, PR 4, PR114, CH 12, CH 15, CH144, LH 54, LH 123, LH 124 y MT21 (Tabla 1). En la fig. 8 aparece un ejemplo de curvas integrales con las gráficas de los pluviómetros al final del texto. 
La primera característica que sobresale en el comportamiento de ciclicidad de la lluvia es que manifiesta un carácter «aperiódico» (duración variable), lo que coincide con el resultado de otros estudios de regularidad climática, realizados por investigadores cubanos y extranjeros, entre los que merecen citarse a: Trusov, et al 1983, Fernández 1988, Fernández y Maximota 1990, Gutiérrez 1993, Lapinel 1997a y Lora 1999a.

En la siguiente tabla se consolida el análisis de las diferentes curvas obtenidas en la figura 8 .

Tabla 8

CICLOS APERIÓDICOS

\begin{tabular}{|c|l|l|l|}
\hline Ciclos & $\begin{array}{l}\text { Duración del ciclo } \\
\text { (No. de años) }\end{array}$ & $\begin{array}{l}\text { Duración de la fase } \\
\text { húmeda.(No. de años) }\end{array}$ & $\begin{array}{l}\text { Duración de la fase seca } \\
\text { (No. de años) }\end{array}$ \\
\hline 1 & $1922-1951$ (29 años) & $1922-1934$ (12 años) & $\begin{array}{l}1934-1951 \\
(17 \text { años })\end{array}$ \\
\hline 2 & $1951-1965$ (14 años) & $1951-1954$ (3 años) & $\begin{array}{l}1954-1965 \\
(11 \text { años })\end{array}$ \\
\hline 3 & $1965-1975$ (10 años) & $1965-1969$ (4 años) & $\begin{array}{l}1969-1975 \\
(6 \text { años })\end{array}$ \\
\hline 4 & $1975-1990(15$ años) & $1975-1983$ (8 años) & $\begin{array}{l}1983-1990 \\
(7 \text { años })\end{array}$ \\
\hline
\end{tabular}

Fuente. Valores representados en las curvas de ciclicidad. Elaborada por los autores.

La duración de cada uno de ellos fue de 29, 14, 10 y 15 años, respectivamente. La regularidad observada es aproximadamente igual a la definida por los especialistas anteriormente mencionados, tanto para el occidente de Cuba como para todo el país, lo que demuestra la concordancia general existente entre sus respectivos resultados.

Cada uno de los ciclos de referencia está compuesto de dos fases, una con características húmedas, denominada «fase húmeda», conformada por un conjunto de años de valores relativamente altos, que es sucedida por otra de carácter menos húmedo, comúnmente denominada «fase seca», conformada por otro conjunto sucesivo de años de valores más bajos. Las fases correspondientes a los cuatros ciclos definidos con anterioridad en el actual siglo, son los siguientes:

Fases húmedas: 1922-1934, 1951-1954,1965-69 y 1975-1983

Fases secas: 1934-1951, 1954-1965, 1969-1975 y 1983-1990

análisis efectuado resultaron cuatro ciclos o períodos multianuales: 1922-1951, 1951-1965, 1965-1975 y 1975-1990.

Por su parte las cimas o momentos (entiéndase fechas) en los que se produjeron cambios de tendencia húmeda a seca, según reportan la mayoría de las curvas construidas, son aproximadamente 1934, 1954, 1969 y 1983 mientras que los momentos inversos o de inflexión más baja fueron 1951, 1965, 1975 y 1990, años que corresponden al momento final de estas curvas, equivalentes a los puntos de cambio de fase seca a fase húmeda. 


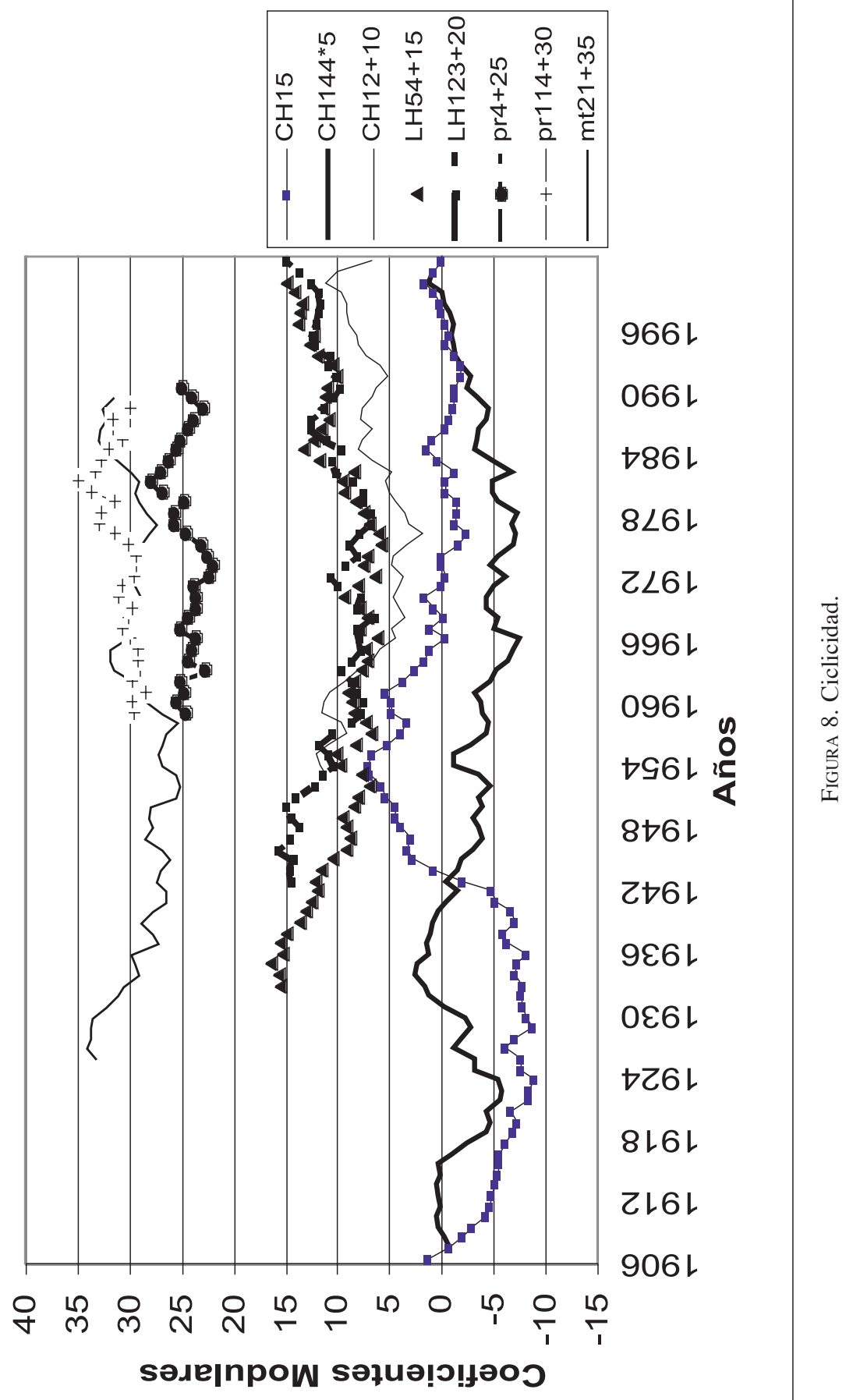


Además de la información antes expuesta se debe mencionar que en el inicio de la serie de datos, 1907 para el CH 15 y 1909 para el CH 144, se presenta (ver figura 8) parte de un ciclo que debió comenzar antes de 1907 (en la gráfica sólo se manifiesta la rama seca de este ciclo). En el gráfico se observa que el cuarto ciclo culmina en 1990, y se manifiesta en él que el ciclo actual no ha concluido.

\section{Conclusiones}

- La estacionalidad de los valores de la lluvia mensual es significativa, presenta una distribución «bimodal» con un máximo primario en el mes de junio y otro secundario en septiembre.

- La variabilidad es muy alta en los meses del período lluvioso. No existe una acumulación de los valores de mayor variabilidad al principio o al final del período estudiado (1961-1993).

- Hay una estacionalidad dinámica expresada por los cambios de los índices del patrón estacional en los meses de febrero y marzo, en los que parece estar cambiando sus valores promedios, por efecto de procesos naturales.

- El comportamiento decadal de la lluvia no arrojó diferencias sustanciales entre las décadas.

- La tendencia anual en el período 1961-1993 es negativa. La lluvia tiende a aumentar en el período poco lluvioso y a disminuir en el período lluvioso. Aunque no en todos los pluviómetros la tendencia fue significativa, sí se manifiesta una alta frecuencia de tendencias negativas en junio, septiembre y octubre, lo que produjo que la tendencia anual arrastrara este signo.

- La ruptura de la tendencia se produce en los años 1973 y 1975, en este último año con mayor precisión.

- Del análisis efectuado resultaron cuatro ciclos o períodos multianuales: 1922-1951, 1951-1965, 1965-1975 y 1975-1990. La duración de cada uno de ellos fue de 29, 14, 10 y 15 años respectivamente. Las fases correspondientes a los cuatros ciclos definidos en el siglo que terminó son las siguientes:

Fases húmedas: 1922-1934, 1951-1954, 1965-69 y 1975-1983

Fases secas: 1934-1951, 1954-1965, 1969-1975 y 1983-1990

\section{5. bibliografía}

CANSADO, E. (1966): Curso de Estadística General. Ediciones Revolucionarias. La Habana. Cuba. $662 \mathrm{p}$.

CARMEJO-RODRÍGUEZ, M. P. (1990): Aspecto oceanográfico y meteorológico del fenómeno El Niño (1986-1987), en Acta oceanográfica del Pacífico INOCAR. Ecuador, 6 (1). DESER, C. y WALLACE, J. M. (1987): «El Niño. Events and their relation to the southern oscillation, 19251986», en Journal Geohysical Research. Volm 92. No c1. 1 4. pp. 18-30.

FERNÁNDEZ, N. (1988): «Formación y cálculo del escurrimiento en los ríos de las provincias orientales de Cuba». Tesis de Doctorado. Universidad Estatal de Moscú. 82 p.

FERNÁNDEZ, N. y MAXINOVA, O. (1990): «Algunas experiencias de los parámetros de la precipitación anual» en Revista Voluntad Hidráulica. Ciudad de La Habana, no 83, pp. 41-47.

FREUND, J.E. (1977). «Estadística Elemental Moderna». Editorial Pablo y Educación. La Habana. Cuba. 466 p.

GUTIÉRREZ, J.E. (1993). «Regularidades espaciales y temporales de los recursos hídricos del occidente de la Isla de Cuba. Tesis de Doctorado. Facultad de Geografía. Universidad de La Habana. Cuba. 158 p. 
HERNÁNDEZ, J. M. (1981). «Investigación Empírica sobre los Métodos de Pronóstico Utilizados por la DDI» en Revista Estadística $N^{o}$ 6. Ciudad de La Habana. Cuba. pp. 9-37.

LAPINEL, B. (1997). «Consideraciones sobre el comportamiento de la lluvia en Cuba en los años más recientes», en Forum de Ciencia y técnica. Centro Meteorológico de Camagüey. Cuba.

LORA, B. (1999). «Estudio preliminar de tendencia de las precipitaciones». Instituto Nacional de Recursos Hidráulicos. La Habana. Cuba. Inédito. 8 p.

Mapas sinópticos. Archivo de la Biblioteca. Instituto de Meteorología. CITMA. Inédito.

Resumen de las temporadas invernales. Dpto. de Pronóstico. Instituto de Meteorología. CITMA, La Habana. Cuba. Inédito.

Resumen de la temporada ciclónica por años. Dpto. de pronóstico. Instituto de Meteorología. La Habana. Cuba. Inédito.

TRUSOV, I. I., IZQUIERDO A., y DÍAZ, L.R. (1983). «Características espaciales y temporales de las precipitaciones atmosféricas». Editorial Academia. Ciudad de La Habana. Cuba. 150 p.

WEI, W., (1990). «Time serie analysis» (Univariante and Multivariante Methods). Addison - Wesley. New York. EE.UU. 400 pp.

WILLKS, D. (1995): «Statistical Methods in the Atmospheries Sciences. An introduction». Academic Press. New York. EE.UU. 500 p. 